\title{
Spatial phase light modulator based on the ferroelectric liquid-crystals: possibility of the device construction
}

\author{
Svetlana Kotova ${ }^{1 *}$, Aleksandra Mayorova ${ }^{1}$,Evgeny Pozhidaev ${ }^{2}$, and Sergey Samagin ${ }^{1}$ \\ ${ }^{1}$ Lebedev Physical Institute RAS, Samara Branch, 221 Novo-Sadovaya Str., Samara, 443011 Russia \\ ${ }^{2}$ Lebedev Physical Institute RAS, 53 Leninskiy Prospekt, Moscow, 119991 Russia
}

\begin{abstract}
The work investigates the possibility for building of zonal spatial phase light modulators employing the quadratic electro-optic effect in ferroelectric liquid-crystals and capable of providing modulation frequencies up to $1 \mathrm{kHz}$. The study is made through the use of numerical simulation methods and experimental modulation characteristics
\end{abstract}

One of important challenges of modern optics is to increase the speed of control of the spatial characteristics of light fields. This task is relevant both to traditional areas of optics, including adaptive optics and holographic storage devices, and for such intensively developing fields of science and technology as holographic displays, optical manipulation of microscopic and nano-scale objects; quantum cryptography; quantum computation. As a rule for this the devices capable of pure phase modulation of light are needed. The traditional way of implementing a pure phase spatial modulation consists in the use of orientation effects in nematic liquid crystals (LC), for which characteristic frequencies are several tens of Hz. Such performance (response speed) restricts the possible scope of application for spatial light modulators (SLM) based on nematic LC. It is possible to increase in performance due to the orientation effects in ferroelectric liquid crystals (FLC) with sub-wavelength pitch of the spiral structure, in particular, orientational Kerr effect $[1,2]$.

Under this work the possibility of constructing spatial modulators of different types based on the cell with a ferroelectric liquid crystal with sub-wavelength pitch of the spiral and with the helicoid axis orientated along the substrates, is analyzed by the numerical simulation method.

As soon as electric voltage is applied to the standard FLC cell with planar orientation of the main axis of the helix, then the change of the optical axis of the FLC layer direction and refractive indexes occurs. The change of the position of the optical axis of FLC in the plane perpendicular to the incident light wave direction causes the change in the state of polarization of the transmitted light. In this paper we studied the possibility of formation of light fields with the required spatial parameters in such conditions. Special attention was paid to the formation of light fields in the form of specified curves possessing orbital angular momentum.

Corresponding author: kotova@,fian.smr.ru 
Electro-optical characteristics of FLC 587 were measured. Approximation of the experimental data allowed us to obtain required for the simulation of the SLM modulator based on FLC, dependencies of refractive indices, birefringence, effective phase delay on the value of the electric field applied to the layer.

Multi-pixel modulators with a square configuration of pixels and sector type modulators operating both in transmission and reflection were considered. Calculations of the light fields were made for the following scheme. The SLM was lightened with the linearly polarized light beam of homogeneous intensity. The value of the voltage fed to each pixel was determined in accordance with the required value of the effective phase delay which should be formed for the transmitted wave. The light transmitted or reflected by the modulator (depending on the modulator type) passed through the polarizer with the plane of transmission coinciding with the initial direction of polarization and was focused by the lens.

The light fields with nonzero orbital angular momentum and the distribution of intensity in the form of ring, curve (Archimedes spiral) and a closed curve (triangle) were obtained. Figure 1 shows an example of the formation of axially symmetric fields with the topological charge of 3 by means of a modulator of the sector type (12 sectors, the FLC layer thickness of $50 \mu \mathrm{m}$, diameter $1 \mathrm{~cm}$ ). For this case and the other ones it was found that the total energy losses reached approximately $11 \%$, the average deviation of the intensity did not exceed $5 \%$.

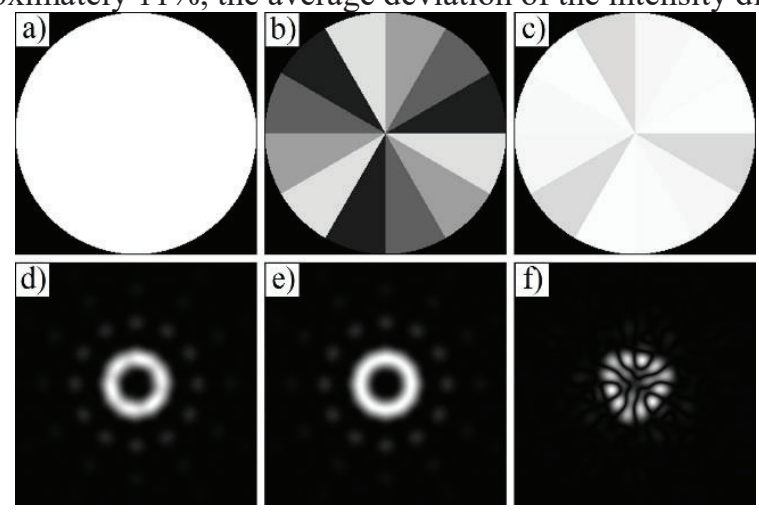

Fig. 1. a) initial distribution of the light field amplitude, b) phase delay profile, c) amplitude of the light field obtained by the polarizer, d) intensity distribution in the focal plane, e) required intensity distribution (without amplitude modulation), f) deviation of the obtained intensity distribution from the desired normalized by its maximum value.

Thus it can be concluded that the resulting amplitude modulation has no significant effect on the formation of light beams with nonzero angular momentum in the form of specified curves. The results of the study indicate the possibility of constructing phase SLM-based FLC with modulation frequencies above $0,5-1 \mathrm{kHz}$.

The research is carried out under the financial support of RFBR (projects No. 16-42-630773 and 1629-14012 ofi-m).

\section{References}

[1] E.P Pozhidaev et al., Phys. Rev. E 87, 052502 (2013)

[2] S.P. Kotova et al., Phys. Rev. E 92, 062502 (2015) 